\title{
Erythematosquamous Dermatosis
}

National Cancer Institute

\section{Source}

National Cancer Institute. Erythematosquamous Dermatosis. NCI Thesaurus. Code C34591.

A skin condition that primarily affects the scalp and face and presents as scaly inflammation. Examples include itchy, dry skin and dandruff. 